nehmen selbst geredet wird, so dürftig ist oft die dahinterstehende Substanz. Der Bochumer Innovationsforscher Erich Staudt kommentierte bereits 1985 das Innovationsverhalten zahlreicher deutscher Unternehmen so: „Man betreibt Forschung und Entwicklung, nur weil die Konkurrenz das auch tut. Man subventioniert Innovationsförderungsprogramme und puscht neue Technologien, nur weil die USA oder Japan dies auch tun usw. Der technokratische Aktivismus verstärkt sich selbst, hält seine eigene Hektik für zunehmende Umweltdynamik und schafft doch keine Beschleunigung, sondern verstärkt Widerstände."

Tatsächliches strategisches Innovationsverhalten der Unternehmen wäre von anderer Art. Es ließe sich als funktions- und sinnorientierte Unternehmenspolitik beschreiben. Funktionsorientierung meint entsprechend ihrer Begründung durch den verstorbenen österreichischen Systemtheoretiker Erich Jantsch, daß Produkte kommen und gehen, (gesellschaftliche Versor-
gungs-)Funktionen aber bleiben. Kein deutsches Automobilunternehmen scheint gegenwärtig gewillt, strukturkonservative Produktpolitik zu überwinden und sich strategisch einem funktionsorientierten Denken zu öffnen (insofern wäre es für den Noch-VW-Markenvorstandsvorsitzenden Goeudevert sicher besser, er ginge von selbst und alsbald).

Sinnorientierung hieße in einer demokratischen Gesellschaft, den tieferliegenden Sinn von Gütern und Dienstleistungen auch sinnhaft zu kommunizieren. Was gegenwärtig nicht nur in der Automobilindustrie praktiziert wird, basiert kommunikativ auf der Produktion falscher Erlebniswelten, operativ auf dem Rückgriff auf Rezepte des Frühkapitalismus und strategisch auf der höchst intelligenten Devise „Augen zu und durch". Wie der Münchner Unternehmensberater Günter Ogger kürzlich in seinem informativen Buch ,Nieten in Nadelstreifen" darlegte, hat all dies viel damit zu tun, daß die deutschen Topmanager in vielerlei Hinsicht eher eine sich geschickt abschließende Kaste sind, als daß sie sich marktwirtschaftlichen Prinzipien gemäß tatsächlich durch Erfolg legitimieren müßten. Ach ja, der Titel dieses Beitrags?! Auf dem Weg ins ökologische Jahrhundert (so freilich die These von Ernst-Ulrich von Weizsäcker in seinem hauptsächlich sehr verdienstvollen Buch ,Erdpolitik $^{6)}$ ) sind wir heute ebenso wenig wie bis vor wenigen Jahrhunderten auf dem Weg ins ewige Himmelreich. Kant bleibt weiter im Recht: die Freiheit des Menschen bleibt abhängig von seiner Bereitschaft zum Aufbruch aus selbstverschuldeter Unmündigkeit. Und zur selbstverschuldeten Unmündigkeit gehören Verweigerungen der notwendig anderen $\mathrm{Zu}$ kunft ebenso wie Verklärungen der Zukunft. Unternehmenspolitik hat in den nächsten Jahren mehr denn je mit Kommunikation zu tun, und das heißt seriöser- wie anständigerweise: mit Aufklärung.

Reinhard Pfriem, Oldenburg

\title{
Umweltpolitik unter veränderten politischen Rahmenbedingungen
}

\section{Phasen der bundesdeutschen Umweltpolitik}

Die Entwicklung der bundesdeutschen Umweltpolitik wurde bislang in drei Phasen eingeteilt: 1. Die Etablierungsphase mit Beginn der sozialliberalen Koalition 1969: In dieser Phase wurden die maßgeblichen Ziele der Umweltpolitik formuliert, die Umweltschutzkompetenzen des Bundes erweitert, Umweltschutzgesetze formuliert und erweitert (BImSchG, Abfallgesetz, WHG, Bundesnaturschutzgesetz etc.).

-2. Die Abschwungphase. Diese Phase, in die die mit den sogenannten Ölpreisschocks einhergehenden rezessiven Tendenzen fallen, verschafte dem Argument Gehör, daß Umweltschutz ein Wachstumshemmnis darstelle und damit Arbeitsplätze gefährde. Folge dieses Meinungswandels war das Zurückstellen und auch Verwässern von Umweltgesetzen (Konferenz von Gymnich).

3. Die sogenannte Konsolidierungsphase ( $a b$ Beginn der achtziger Jahre), die letztlich durch den ökologischen Handlungsdruck - Stichwort Waldsterben - und vor allem auch durch die Umweltverbände, Bürgerinitiativen und auch die GRÜNEN erreicht werden konnte. Insbesondere wurden Umweltschutzauflagen als Wachstumshemmnis anders eingeschätzt, ausgelöst durch eine Studie im Auftrag des BDI. In der Folge wurde dann die Auffassung vertreten, daß umweltfreundliche Technologien sich selbst zu einem Wachstumsmotor entwickeln könnten, d.h. von einem Jobkiller zum Jobknüller werden könnten. In diese Phase fallen auch die Programme Arbeit und Umwelt, die insbesondere von der SPD als explizite Be- schäftigungsprogramme in verschiedenen Bundesländern aufgelegt wurden. Zudem wurden eine Vielzahl rechtlicher Regelungen im Umweltbereich durchgeführt, wovon u. a. die Großfeuerungsanlagenverordnung einen der wesentlichsten Einflüsse auf die spürbaren Umweltentlastungen hatte.

\section{Eine neue Phase der Umweltpolitik?}

Eine neue Zäsur, möglicherweise eine neue Phase der Umweltpolitik, deutet sich seit Beginn der 90 er Jahre an. Es existieren hier aber sehr unterschiedliche Orientierungen:

- Nach Ansicht von Umweltminister Töpfer tritt die Umweltpolitik in eine neue Phase ein, die gekennzeichnet ist durch die Stärkung des Verursacher- bzw. Vorsorgeprinzips und die Einführung der Gefährdungshaftung. Desweiteren sei im Bereich der Abfallwirtschaft der erste Schritt zu einer Kreislaufwirtschaft getan, und die integrierten Technologien würden immer mehr an Bedeutung gewinnen - mithin stehe ein Ergrünen der Wirtschaft bevor. Dem ist insoweit zuzustimmen, als zwar versucht wird, weitgehende gesetzliche Regelungen zu erlassen. Der aktuelle Stand deutet allerdings eher darauf hin, daß eben diese Orientierungen letztlich verwässert wurden, z. B. durch das im $\mathrm{Be}-$ reich der Abfallwirtschaft in aller Regel vorgegebene Primat der Wirtschaftlichkeit oder auch, wie aktuell deutlich wird, in der Verschiebung von Abfallproblemen (Abfall als Wertstoff) ins Ausland.
- Nach Ansicht führender Wirtschaftsorganisationen stellt sich allerdings die Frage, inwieweit nicht die Belastungen aus der Umweltgesetzgebung in der Bundesrepublik bereits zu hoch seien und insbesondere in Zeiten schwieriger wirtschaftlicher Situationen keine Vorreiterrolle im Umweltschutz eingenommen werden könne. Aus dieser Orientierung scheint die Umweltpolitik sich eher wieder auf dem Weg in eine Stagnationsbzw. Abschwungsphase zu befinden, in der zwar die ökologischen Problemlagen bekannt und benannt sind, die wirtschaftlichen und politischen Kräfteverhältnisse jedoch eine Forcierung der Umweltpolitik in Frage stellen. Die Forderung nach einer vermeintlich notwendigen Pause in der Umweltpolitik wird von Teilen der Gewerkschaften, der Unternehmerverbände und auch von Politikern offensiv vertreten.

Unabhängig von der Einschätzung der weiteren Orientierung der Umweltpolitik bleibt der ökologische Handlungsdruck hingegen hoch und das Spannungsverhältnis zwischen dem, was wirtschaftlich möglich und dem was ökologisch notwendig ist, erhöht sich. Damit stellt sich die Frage, ob sich die Abschwungphase der Umweltpolitik der 70er Jahre wiederholt oder ob der existierende ökologische Problemdruck zu einem aktiven umweltpolitischen Eingreifen führt.

\section{Eine kurze Bilanz \\ der bisherigen Umweltpolitik}

Die vorzeigbaren Ergebnisse der bundesdeutschen Umweltpolitik - sie werden insbesondere im Vergleich zu den neuen Bundesländern sowie Mittel- und Osteuropa dargestellt, - lassen sich in starkem Maße additiven Technologien zuordnen. Dies in dem Sinne, 
- daß es zu Verschiebungen der medialen Belastungen kommt, z.B. von der Luft- oder auch Wasserverschmutzung zur Abfallproblematik,

daß diese Erfolge z.T. durch weiteres wirtschaftliches Wachstum wiederum in Frage gestellt werden,

- daß es sich entsprechend bei den Entkoppelungstendenzen um zeitlich begrenzte Phänomene handelt und

zudem eine Verlagerung festzustellen ist von den sogenannten altindustriellen hin zu den dienstleistungsorientierten Belastungen (Verkehr, Flächenverbrauch etc).

Die umweltpolitischen Erfolge werden somit zumindest auf zwei Ebenen konterkariert:

- Durch andere Politikbereiche, sei es durch die Landwirtschaftspolitik, die Energiepolitik, die Wohnungsbaupolitik etc. Gerade in diesem Zusammenhang ist es bedeutsam, daß die fortschrittliche Umweltpolitik eben durch andere Politikbereiche in Frage gestellt wird.

- Durch das Wirtschaftswachstum, das gekennzeichnet ist durch zusätzliche Umweltbelastungen. Dazu sei die Studie von Martin Jänicke Umweltentlastung durch Strukturwandel? in Erinnerung gerufen und die darin gemachten Aussagen, die verdeutlichen, daß Entkopplungstendenzen zwischen Umweltverbrauch und Wirtschaftswachstum nur partiell und meist nur über gewisse Zeiträume erreicht werden können, daß danach sich aber eher wieder gleichgerichtete Entwicklungen ergeben.

Der zentrale Punkt der bisherigen Umweltpolitik ist der, daß sie zum einen nur begrenzte Erfolge hatte und zum anderen, daß sie niemandem wirklich weh tat, die Verteilungskonflikte sich insofern in Grenzen hielten. Die Umweltprobleme verursachende Wachstumswirtschaft ist dabei die Problemlöserin für bestimmte Probleme des Umweltschutzes und zugleich der Lösungsmechanismus für ökologische Verteilungsprobleme. Daher muß gefragt werden, inwieweit dieser Mechanismus in Osteuropa bzw. weltweit greifen kann, oder ob es sich dabei nicht vielmehr um Möglichkeiten von entwickelten Industriegesellschaften handelt, in denen lediglich die technischen Verbesserungsmöglichkeiten und die ausschöpfbaren Effizienzsteigerungen genutzt wurden, eine umfassende Begrenzung der Umweltschädigungen hingegen kaum erreicht wurde. So werden gerade diese Effizienzerfolge des westlichen Wirtschaftssystems hervorgehoben, wobei als Maßeinheit die Emissionen bzw. der Energieeinsatz je Produkteinheit betrachtet wird; die Betrachtung der absoluten Höhe unterbleibt aber in der Regel.

\section{Kennzeichen}

\section{für eine neue Umweltpolitik}

Ein Kennzeichen einer neuen Phase der Umweltpolitik könnte es sein, daß die Umweltpoli- tik wegkommt von der Emissionsfixierung, die in der Regel mit nachgeschalteten Technologien verbunden ist, und Schritte auf die Betrachtung und Bewertung von Stoffströmen im Sinne einer integralen Betrachtungsweise vornimmt. Dabei scheint die Frage der $\mathrm{CO}_{2}$-Problematik für die Umweltpolitik ein in diesem Sinne zentraler Gradmesser zu sein. Dies ist darin begründet, daß hier Verschiebungen und Verlagerungen gegenwärtig technisch nicht durchführbar sind, insofern keine aus der Umweltpolitik hinreichend bekannten Ausweichstrategien bzw. technokratischen Lösungen gefahren werden können. Es existieren für die Lösung dieses Problems (unter Ausklammerung der Kernenergie) im wesentlichen zwei Optionen:

- die Effizienzoption,

die Veränderung des Wohlstandsmodells (bzw. die Verzichtsoption?).

Beide Strategien sind gangbar, wobei die Effizienzoption kurzfristig erhebliche Entlastungspotentiale eröffnet, langfristig nach Ausschöpfung der einfach erschließbaren Potentiale hingegen kostenintensiv wird. Entscheidend ist allerdings, daß die Effinzienzerfolge nicht durch Wirtschaftswachstum in Frage gestellt werden. Die gesellschaftliche Orientierung auf ein $\mathrm{Zu}$ rückfahren der Stoffumsätze (was auch unter der Rubrik neues Wohlstandsmodell verzeichnet werden kann) bedeutet hingegen, daß es zentral auch um die Verteilungsproblematik gehen wird.

Letztendlich sind beide Optionen aber nicht getrennt voneinander zu betrachten, denn eine alleinige Orientierung auf die Effizienzoption dürfte hinsichtlich der Reichweite der Strategie nicht ausreichend sein. Zudem stellt eine weitgehende Erschließung der Effizienzmöglichkeiten eine gewisse Annäherung an die Verzichtsoption dar, da aufgrund der steigenden, hohen Kosten zwangsläufig entsprechende Reaktionen erfolgen.

\section{Zur Frage der Standortfaktoren -} Aspekte einer aktuellen Diskussion

Fragen nach dem Unternehmensstandort sind in der Regel geprägt durch eine Vielzahl von höchst unterschiedlichen Faktoren, von denen ein wichtiger die Umweltgesetzgebung bzw. die Umweltauflagen eines Landes ist. Dabei ist die Frage zentral, welche Auswirkungen die Umweltpolitik auf die unterschiedlichen Sektoren der Wirtschaft hat. Umweltminister Töpfer hat ein Gutachten erarbeiten lassen, in dem die Standortfrage untersucht wurde. Im folgenden seien einige Aspekte aus diesem Gutachten aufgeführt:

- Der zentrale Indikator für die Standortbedingungen sind in der Regel die Unternehmensrenditen. Diese sind nach einer Untersuchung des Instituts der Deutschen Wirtschaft, einem wirtschaftsnahen Forschungsinstitut, in der Bundesrepublik in Relation zu anderen Industriestaaten niedrig. Für Investitionsentscheidungen selbst ist die Verzinsung des eingesetzten Eigenkapitals wichtiger; hier besetzt die Bundesrepublik ebenfalls einen der hinteren Plätze.

- Der Saldo der Direktinvestitionen in das Ausland ist erheblich angestiegen, wobei sich im Bereich der Industrie insbesondere solche Unternehmen in das Ausland orientierten, die selbst stark exportorientiert sind. Zugleich ist festzustellen, daß ausländische Investoren nicht im selben Umfang in Deutschland investiert haben.

Gemessen am Anteil am BSP ist festzustellen, daß die Umweltschutzausgaben in der Bundesrepublik im Vergleich zu den meisten anderen Industriestaaten seit 1980 gestiegen sind. Mit einem Anteil von 1,7\% am BSP liegen die Ausgaben in der Bundesrepublik zwar hoch, allerdings ist der Abstand zu anderen Ländern nicht sehr groß.

- In der Untersuchung werden die umweltintensiven Wirtschaftsbereiche hinsichtlich der durchschnittlich getätigten Gesamtaufwendung definiert. Diese Auflistung gilt auch dann, wenn sie mit bestimmten Schadstoffindikatoren in Verbindung gesetzt werden. Danach gelten die folgenden Wirtschaftsbereiche als besonders umweltintensiv: Energieund Wasserversorgung, Bergbau, Mineralölverarbeitung, NE Metallerzeugung, Chemische Industrie, Eisenschaffende Industrie, Zellstoff- Papier, Pappeerzeugung, Gießereien, Holzbearbeitung und Steine/Erden.

$\mathrm{Zu}$ berücksichtigen ist allerdings, wie die genannten Produkte wiederum in anderen Wirtschaftssektoren verwendet werden. Damit stellt sich die Frage nach den direkten und den indirekten Kosten des Umweltschutzes. Dies läßt sich mit Hilfe der Input-Output-Analyse unter der Restriktion durchführen, daß alle Wirtschaftsbereiche die von ihnen aufgewendeten Kosten des Umweltschutzes in voller Höhe auf ihre Abnehmer abwälzen und die Struktur der Umweltschutzaufwendungen weitestgehend der Struktur der Schadstoffemissionen entspricht: Auch in diesem Falle ändert sich an der Zusammensetzung der Branchen wenig, allerdings wird deutlich, daß einigen Investitionsgüterbranchen die hohen Umweltschutzaufwendungen der Vorlieferanten zurechenbar sind und jene Branchen damit auch entsprechend umweltintensiv sind. Die Diskussion um die Umweltstandards wird in starken Maße von den Industriezweigen geprägt, die aus der oben angeführten Gruppe stammen.

\section{Aussicht und Perspektiven}

- Die politischen Entwicklungen in Osteuropa gewinnen für die westeuropäischen Staaten immer mehr an Bedeutung, da das ungeheure ökonomische und Wohlstandsgefälle zwischen Ost und West ohne die Errichtung von Mauern auf Dauer nicht zu halten ist. Dies bedeutet dann letztlich auch, die Frage zu stellen, inwieweit die Teilungsbereitschaft in den westeuropäischen Staaten vorhanden ist. 


\section{Bemerkung zum Zusammenhang der steigenden Arbeitsteilung mit dem Verkehrsaufwand*}

Der Umbruch in Europa führt zu einem Prozeß steigender Arbeitsteilung. Eine der in ihrem Umfang am wenigsten bedachten Folgen ist das enorm steigende Verkehrsaufkommen. Der folgende Beitrag leitet theoretisch $a b$, wie immens die Steigerungsraten des Transports tatsächlich sein werden - im Gegensatz zu den Aussagen einiger der mit diesem Thema befaßten Ökonomen.

Es wird in den jüngsten Debatten um die weitere Entwicklung des Verkehrsaufwandes in Europa immer wieder angenommen, die Steigerungsraten insbesondere des motorisierten Straßengüterverkehrs würden von Experten überschätzt, teilweise werden sogar die äußerst maßvollen Voraussagen etwa des PROGNOSInstituts (40\% mehr bis 2005) als überhöht bezeichnet. Insbesondere wird teilweise vehement negiert, daß die weiter steigende Arbeitsteilung überhaupt nennenswerten Einfluß auf den Transport hat (vgl. etwa entsprechende Ausführungen von Dieckmann 1992, S. 256, der unter dem Absatz „Vorurteile beherrschen die Diskussion" entsprechende Einflüsse abtut).

$\mathrm{Zu}$ dieser Frage sollen im folgenden einige prinzipielle Überlegungen angestellt werden, welche der Dynamik des Prozesses Arbeitsteilung/ Verkehr näherzukommen versuchen.

Bei fast allen produktiven Abläufen wird in der jüngsten Zeit die Arbeitsteilung dadurch erhöht, daß die sogenannte „Fertigungstiefe“" (das ist der Anteil an einem Produkt, der von einem Unternehmen selbst hergestellt wird), weiter verringert wird. So haben große Autohersteller (in Deutschland etwa Opel oder VW) längst nur mehr eine
Fertigungstiefe unter $25 \%$. Selbst bei einem scheinbar so simplen Produkt wie einem Joghurt für den Frühstückstisch hat mittlerweile die $\mathrm{Zu}$ liefererkette eine kaum glaubliche Dimension angenommen (vgl. Böge 1992). Der Prozeß ist natürlich dadurch gekennzeichnet, daß die Fertigungstiefe auf jeder Ebene abgebaut wird, auch der Zulieferer des Zulieferes versucht gerade in der gegenwärtigen angespannten ökonomischen Situation, ,abzuschlanken" (,lean-production") und die Produktion von Produktbestandteilen abzugeben, wenn dies irgend rentiert.

Man kann nun den Effekt der Verringerung der Fertigungstiefe modellhaft abschätzen. Es gibt verschiedene Arten der Verhältnisse von einem Endhersteller zu seinem Zulieferer. Abbildung 1 zeigt einige idealisierte und systematisch begrenzte (1) Fälle auf.

Alle drei Fälle zeigen die Produktion eines Produktes auf drei Herstellungsebenen. Nehmen wir an, auf jeder Fertigungsebene würde ein Drittel des Wertanteils des Produktes hergestellt, beträgt die sogenannte Fertigungstiefe auf

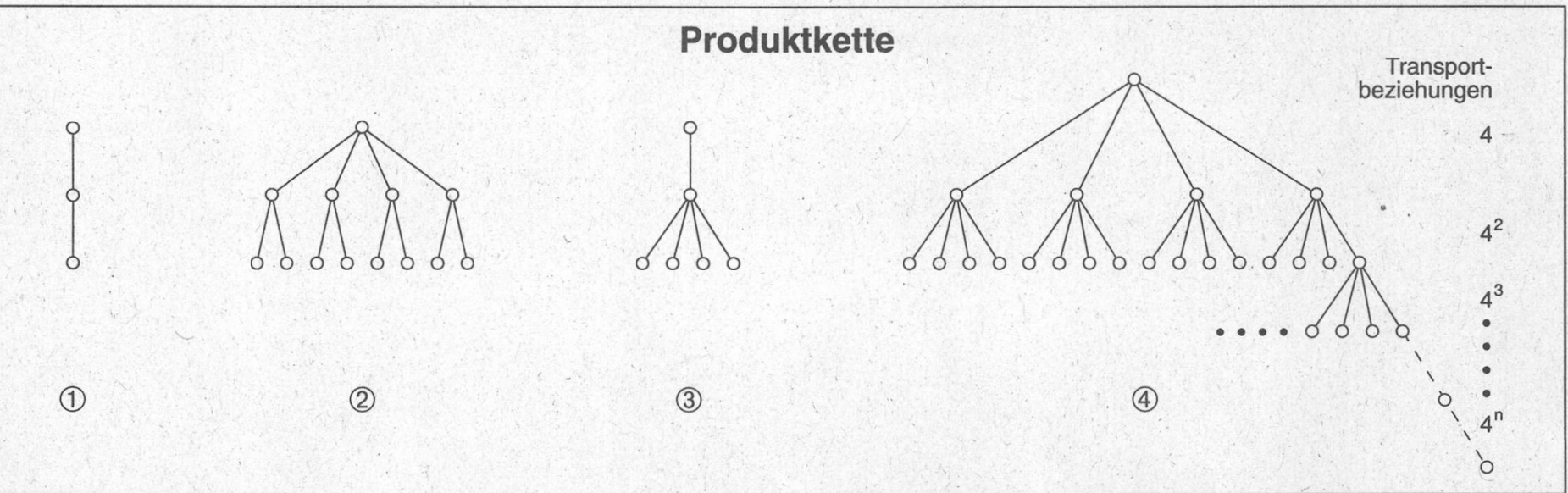

Abbildung 1

- Der Fall der neuen Bundesländer hat deutlich gemacht, welche Finanztransfers notwendig werden, um die Situation der osteuropäischen Staaten an die der westeuropäischen Staaten heranzuführen, ohne daß es kurz- bis mittelfristig gelingen kann, intern an die Leistungsfähigkeit der westlichen Staaten heranzukommen. Nun kann zwar kein direkter Vergleich zwischen der Situation in den osteuropäischen Staaten und den neuen Bundesländern vorgenommen werden; Transfers in diesem Umfang werden nicht möglich sein, zudem ergeben sich auch andere Anforderungen aufgrund der Existenz eines Binnenmarktes und eines anderen sozialen Absicherungssystems. Dennoch wird deutlich, daß über Jahrzehnte mit deutlich anderen Verhältnissen gerechnet werden muß.

- Wiederaufbau der Infrastrukturen in den neuen Ländern sowie die Aufrechterhaltung und Instandhaltung der Infrastruktur in den alten Bundesländern, die auf Dauer erhebliche Mittel erfordern werden. Bereits jetzt wird deutlich, daß es in einigen Bereichen zu einem
Zerfall der Infrastruktur aufgrund der fehlenden Mittel kommen wird, da z. B. die Strukturhilfemittel im Westen weggefallen sind.

- Es muß mit verstärkten finanziellen Engpässen der Staats- und Sozialversicherungshaushalte auf längere Frist gerechnet werden, dies für die Bundesrepublik Deutschland schon alleine aufgrund der hausgemachten Probleme in den neuen Bundesländern. Gründe für die zunehmenden Finanzierungsschwierigkeiten sind u. a. die Arbeitslosigkeit und damit zusammenhängend die Probleme der Finanzierung des "sozialen Netzes“.

Die bisherigen Phasen der deutschen Umweltpolitik haben sich in einem - zumindest aus heutiger Sicht - eher ruhigem Fahrwasser abgespielt. Mit dem angeblichen Eintritt in die sogenannte vierte Phase kommt sie aber in erhebliche politische und wirtschaftliche Turbulenzen, wie die Stichpunkte angedeutet haben. Dies hat zur Folge, daß manch liebgewonnene Perspektive und Orientierung neu übergedacht werden muß.

Ulrich Petschow, Berlin jeder Ebene ca. $33 \%$, im ersten Fall gilt dies für jeden Teilproduzenten. Praktisch dürfte ein solch einfacher Fall der Produktion einer Ware kaum noch existieren. Die Fälle 2 und 3 entsprechen schon eher uns bekannten Formen der Arbeitsteilung, im Falle 2 reduziert sich mit zunehmender Rückverfolgung der Fertigungskette die Anzahl der Zulieferer (von 4 auf 2), im Fall 3 erhöht sie sich und ist beim Zulieferer höher als bei der Endfertigung.

Je nach Produkt wird ein solches Schema (Produktkette) anders aussehen, in den heute üblichen Fällen industrieller Produktion sind jedoch bereits wesentlich mehr Zulieferer als bei den vorgestellten Beispielen üblich. Bei einem Glas Joghurt sind mindestens 12 direkte Zulieferer beteiligt, beim Zulieferer des Glases z. B. sogar mehr als 12 Zulieferer. Wenn im folgenden kurz die Halbierung der Fertigungstiefe analysiert wird und wir dabei von 4 Zulieferern ausgehen, denen wiederum je 4 Zulieferer zuliefern, erscheint dies eher wenig (vgl. Abbildung 2).

Um die Vergrößerung der Zuliefererbeziehungen in einem einfachen Modellansatz abzu- 
schätzen, nehmen wir die Anzahl der Zulieferer auf jeder Stufe als gleich an und führen folgende Größen ein:

ZUL = Anzahl der Zulieferer auf jeder Stufe $\mathrm{N}=$ Anzahl der Produktionsstufen

Bei diesem Modellansatz können wir

ZUL Zulieferer von Stufe 2 nach Stufe 1 $(\text { ZUL })^{2}$ Zulieferer von Stufe 3 nach Stufe 2

und allgemein

$(\text { ZUL })^{k}$ Zulieferer von Stufe k+1 nach Stufe k identifizieren.

Mit der binomischen Fomel lassen sich die $\mathrm{Zu}-$ liefererzahlen auf allen Stufen aufsummieren:

$$
\sum_{i=1}^{N-1}(Z U L)^{i}=\frac{(Z U L)^{N}-1}{Z U L-1}-1
$$

Verkleinert man nun im Modell die Fertigungstiefe und vergrößert damit die Zahl der Produktionsstufen auf N2, so ergeben sich nach obiger Formel

$=\frac{(\mathrm{ZUL})^{\mathrm{N} 2}-1}{\mathrm{ZUL}-1}-1$ Transportbeziehungen.

Der Faktor der Zunahme der Transportbeziehungen bei Verringerung der Fertigungstiefe läßt sich demnach als Quotient beider Sum-

\section{IÖW-Adressen}

\section{Neue Postleitzahlen ab 1. Juli}

\section{IÖW-Geschäftsstelle}

Giesebrechtstraße 13

1000 (10629) Berlin 12

Tel. (030) 882 6094, Telefax 8825439

IÖW-Regionalbüiro Nordrhein-Westfalen

Völklinger Straße 9 ,

5600 (42285) Wuppertal 2

Tel. (02 02) 805 30, Telefax 83402

IÖW-Regionalbüro Schleswig-Holstein

Waitzstraße 11,

2300 (24105) Kiel 1

Tel. (0431) 566362, Telefax 578336

IÖW-Regionalbüro Baden-Württemberg Bergheimerstraße 97 ,

6900 (69115) Heidelberg

Tel. (06221) 167954, Telefax 27060

\section{IÖW-Projektbüro Hannover}

Hausmannstraße 9,

3000 (30159) Hannover

Tel. (05 11) 1640342 , Telefax 854339

IÖW-Österreich, Geschåftsstelle

Rechte Wienzeile 19/5, A-1043 Wien

Tel. (0043-222) 5872189

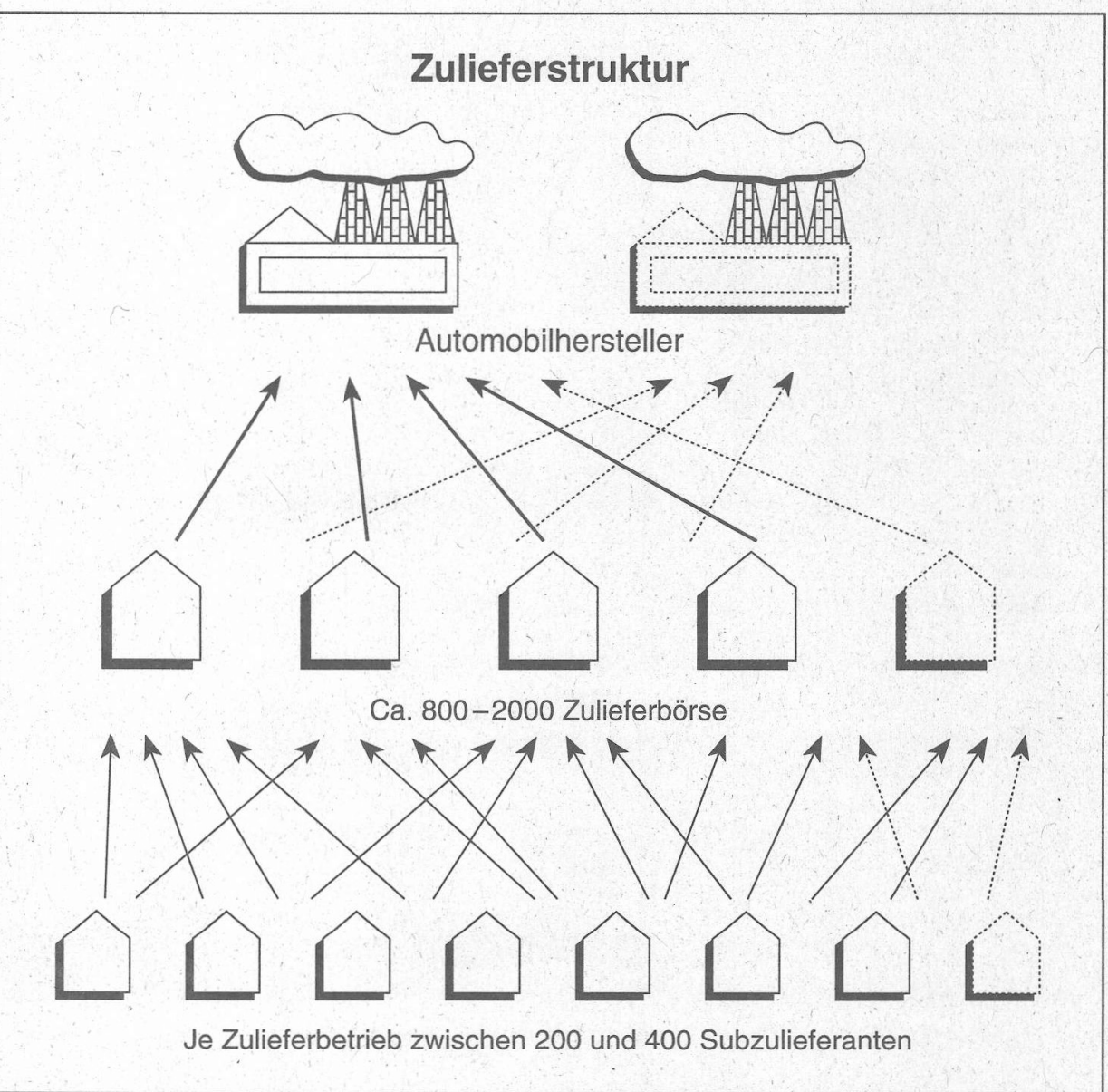

Abbildung 2 (aus Doleschal/Klönne, 1990. S. 12; Originalgrafik: JIT-Graphik)

menformeln beschreiben. Wir nennen diesen Faktor FTrans und schätzen ihn wie folgt ab:

$$
\text { FTrans } \approx \frac{(Z U L)^{N 2}-1}{Z U L-1} / \frac{(Z U L)^{N}-1}{Z U L-1} \approx(Z U L)^{N 2-N}
$$

Eine Halbierung der Fertigungstiefe auf jeder Stufe und eine damit einhergehende Verdoppelung der Fertigungsstufen, also der Fall N2 $=2 * \mathrm{~N}$, ergibt den Faktor FTrans $=($ ZUL $)$ N. Bei $4 \mathrm{Zu}$ lieferern $\mathrm{ZUL}=4$, führt eine Verdoppelung der Fertigungsstufen von 3 (entsprechend $33 \%$ Fertigungstiefe) auf 6 (entsprechend 16,5\% Fertigungstiefe) zu einer Zunahme der Transportrelationen um den Faktor FTrans $=4^{3}=64$. Eine Halbierung der Fertigungstiefe bei 6 Zulieferern ergibt bereit den Faktor FTrans $=6^{3}=216$, und bei $\mathrm{ZUL}=7$ den Faktor 343.

Zwar muß die Erhöhung der Transportrelationen nicht unbedingt eine entsprechend große Zunahme der Transportmenge bedeuten (hier wären produktspezifische Betrachtungen nötig), aus der abgeleiteten Funktionsbeziehung läßt sich aber die generelle These begründen, daß die Verringerung der Fertigungstiefe erheblichen Einfluß auf den Verkehr hat und neue Verkehrsbeziehungen in hohem Maße generiert. Dies gilt insbesondere, wenn die Zahl der Vorproduzenten bereits hoch ist, auf jeder Zulieferebene wieder zahlreiche Zulieferer existieren und wenn die jeweilige Fertigungstiefe bereits gering ist.

Helmut Holzapfel Richard Vahrenkamp Kassel

* Die Grundidee für diese Arbeit entstammt einer Diskussion mit Gisela Hillmann

(1) Betrachtungen der Herstellungsabläufe eines Produktes enden immer an einer gesetzten Limitierung. Etwa das bekannte Joghurt-Beispiel bei der Produktion der Milch beim Bauern. Der aber bezieht wieder Futter für die Kühe aus den USA oder Asien, das dort wieder (unter Düngemitteleinsatz) produziert wird usw. Theoretisch kann man eine solche „Produktkette“ unendlich lang machen. Das beeinflußt jedoch die hier dargestellten Tatbestände nicht. Der unendliche Regress in den Produktionsketten wird am besten mit Input-OutputModellen konzipiert. Die Leontief-Inverse ermöglicht die Aufsummation des unendlichen Regresses.

\section{Literatur}

- Böge, Stefanie 1992: Die Auswirkungen des Straßengüterverkehrs auf den Raum. Die Erfassung und Bewertung von Transportvorgängen in einem Produktlebenszyklus. Dortmund

- Dieckmann, Achim 1992: Marktwirtschaft im Verkehr als Determinate ökologisch und ökonomisch ausgewogener Arbeitsteilung. In: Wirtschaftsdienst 11 , S. 562-565

- Doleschal, Rainer, Klönne, Arno (Hg.) 1990: Justin-time-Konzepte und Betriebspolitik, Hans-Böckler-Stiftung, Bonn

- Holzapfel, Helmut 1992: Der Güterverkehr und die Grenzen der Arbeitsteilung. In: Wirtschaftsdienst 11, S. $555-558$ 
(c) 20I0 Authors; licensee IÖW and oekom verlag. This is an article distributed under the terms of the Creative Commons Attribution Non-Commercial No Derivates License (http://creativecommons.org/licenses/by-nc-nd/3.o/), which permits unrestricted use, distribution, and reproduction in any medium, provided the original work is properly cited. 